\title{
Responses of chromosome segregation machinery to mechanical perturbations
}

\author{
Takeshi Itabashi ${ }^{1}$, Jun Takagi ${ }^{1}$, Kazuya Suzuki $^{1}$ and Shin'ichi Ishiwata ${ }^{1,2}$ \\ ${ }^{1}$ Department of Physics, Faculty of Science and Engineering, Waseda University, Tokyo 169-8555, Japan \\ ${ }^{2}$ Waseda Bioscience Research Institute in Singapore (WABIOS), Singapore 138667, Singapore
}

Received April 9, 2013; accepted May 23, 2013

\begin{abstract}
For genome stability, the proper segregation of chromosomes is required. The exquisite process of chromosome segregation has charmed a lot of cell- and molecular biologists into watching what happens inside a mitotic cell and how each molecule contributes to this process for the accomplishment of accurate cell division ${ }^{1}$. The process to partition the duplicated genome to the daughter cells in each cell division is mediated by a selforganized structure called the mitotic spindle. It is well known that the mitotic spindle is a multi-component macromolecular machine composed of microtubules, molecular motors (kinesins, cytoplasmic dynein), and other regulatory molecules (microtubule-associated proteins, kinases, etc.). In recent years, most of the protein components of the mitotic spindle have been identified and the functions of these proteins have been characterized using molecular perturbations ${ }^{2,3}$. Thus, the mechanisms for spindle assembly and chromosome segregation are being revealed rapidly. However, the chromosome segregation machinery is poorly understood from the mechanical point of view, such as how the mitotic spindle within a cell responds to a variety of mechanical forces, originating from cell-cell interactions or environmental fluctuations. Recent advances in the controlled mechanical perturbation have indicated that the mitotic spindle possesses a structural pliability, size adaptability to the applied external forces, and a strong self-organizing ability. Mechanical perturbations revealed also the mechanochemical regulation of chromosome segregation machinery, which responds to the applied forces. Here, we discuss the current progress in the biophysical
\end{abstract}

Corresponding authors: Takeshi Itabashi, e-mail: t.itabashi@ruri.waseda.jp; Shin'ichi Ishiwata, e-mail: ishiwata@waseda.jp research on the architectural and functional dynamics of the mitotic spindle.

Key words: mitotic spindle, metaphase, cell division, mitotic force, mechanobiology

Dynamic behavior of chromosomes during cell division rests upon the forces generated by the mitotic spindle. The intricate, but well-organized behaviors of mitotic spindle and chromosomes are achieved by the cooperation between various proteins, the basic mechanism of which is conserved across eukaryotes. The mitotic spindle captures condensed chromosomes, aligns them at its center, and pulls apart toward the opposite spindle poles. In metaphase, mitotic spindle has the stable bipolar shape and constant size until chromosome segregation starts, whereas the spindle components, even within the cytoskeletal framework (i.e. microtubules), continuously turn over. It has been suggested that the dynamical stability of a spindle could be regulated by the force balance between the outward force generated by molecular motors and the inward force produced by sister chromatids. After all pairs of sister chromatids simultaneously start the segregation in anaphase, the pole-to-pole distance is elongated by the outward force. In addition, sister chromatids are towed away to spindle poles, resulting in sufficient physical separation to ensure the error-free genetic succession. So the mitotic spindle is an open system that exchanges components (proteins) and energy (ATP) with surrounding cytoplasm to generate force necessary for cell division. However, the micromechanics of the mitotic spindle was poorly understood ${ }^{4}$. 


\section{Mechanical responses of the spindle architecture}

Direct micromanipulation of the self-organized spindle

Mechanical properties of a mitotic cell including an oocyte have been examined by using micromanipulation techniques for a long time ${ }^{5,6}$. Pulling force that acts to separate sister chromatids towards a spindle pole during anaphase was microscopically measured in intact grasshopper spermatocyte using a glass microneedle ${ }^{7}$. One of the classic studies in invertebrate eggs showed structural characteristics of the mitotic spindle in metaphase and anaphase by stretching or compressing it with a pair of microneedles ${ }^{8}$. However, the quantitative mechanical properties of mitotic spindle remained unsolved, since appropriate techniques for the quantitative measurement have not been developed. In addition, the cell membrane hampers direct manipulation of the mitotic spindle inside a cell. To overcome this problem, we have used Xenopus egg extract system, which is a membrane-free system enabling to assemble a large number of spindles in vitro. In the cytoplasmic extract fractionated from eggs, metaphase spindles with average length (the distance between spindle poles) of ca. $37 \mu \mathrm{m}$ are formed spontaneously (Fig. 1a).

Manipulating devices such as a micro-fabricated cantilever $^{9}$ or a glass microneedle ${ }^{10}$ are useful for the direct micromanipulation of metaphase spindle self-organized in such an in vitro system. Under the fluorescence microscope, a pair of either glass microneedles or cantilevers is used to hold a spindle floating in the cytoplasmic extract. The movement of manipulating devices is precisely controlled by the piezo-actuator so that we can quantitatively describe the compressing/elongating force applied to the metaphase spindle. Compared to chemical or molecular biological perturbations, e.g., RNA interference or microtubule-targeted drugs, this mechanical perturbation enables us to modulate reversibly and arbitrarily the force balance within the spindle without any changes in the cytoplasmic solution.

\section{Mechanical responsiveness of the metaphase spindle}

The mechanical properties of metaphase spindles were examined by compressing them in directions either perpendicular or parallel to the spindle pole-to-pole axis. The analysis of mechanical properties and deformability of metaphase spindle showed different mechanical responses, depending on the duration and extent of applied force. Metaphase spindles prepared in Xenopus egg extract show viscoelastic response to small deformations (for example, $10 \%$ compression of spindle width) applied by a pair of cantilevers (Fig. 1b). Recently, the molecular basis accounting for the dynamic micromechanics of an internal spindle structure was further elucidated by combining glassmicroneedle techniques with molecular perturbation of key spindle components ${ }^{10}$; microtubule rigidity and crosslinking by kinesin-5 and dynein are responsible for spindle viscoelasticity. On the other hand, larger (for example, $40 \%$ (a)

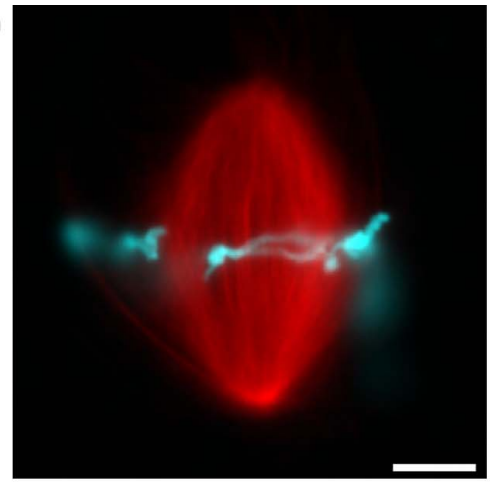

(b)

(c)
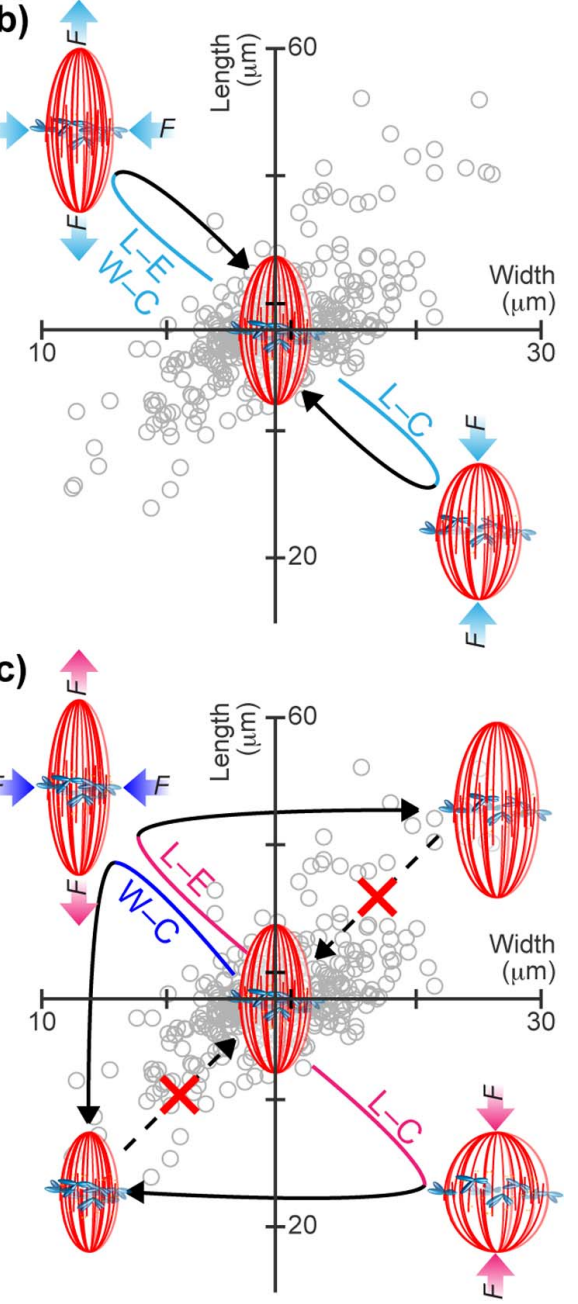

Figure 1 Mechanical responses of a meiotic spindle. (a) Fluorescent image of a mitotic spindle assembled in Xenopus egg extract. Microtubules are shown in red, and chromosomes in blue. Scale bar, $10 \mu \mathrm{m}$. (b) Viscoelastic responses of a meiotic spindle. Meiotic spindles almost recover the initial shape and size after the small or transient mechanical perturbations. (c) Plastic deformation and shape recovery of a meiotic spindle. Larger or persistent mechanical perturbations induce plastic deformation of the spindle. Deformed spindle recovers the initial shape, but is either smaller or larger, depending on the direction of the applied force. Gray circles represent the size distribution of spindles self-organized in Xenopus egg extract. The average length and width were, respectively, 37 and $19 \mu \mathrm{m}$. L-C, length compression; L-E, length elongation; W-C, width compression. 
compression of spindle width) and/or longer (the order of several minutes) mechanical perturbation resulted in plastic deformation, which over several minutes established a structure of smaller size but with elastic properties similar to unperturbed spindles (Fig. 1c). This reorganization could be caused by the adjustment of microtubule dynamics, which responds to and adapts to the persistent application of the external force ${ }^{11}$. Such a structural pliability and high self-organizing ability may be important to achieve faithful chromosome segregation.

Current models of how the size of a spindle is determined focus on the interplay between the diffusible signals that are generated at chromatin and activities of motor proteins. Signals generated by chromosomes include gradients of GTPase activities ${ }^{12,13}$ and phosphorylation of microtubuleassociated proteins ${ }^{14,15}$. These signals have been suggested to be important for controlling the size of spindles selforganized in vivo and in vitro ${ }^{16,17}$. Our experiments demonstrated, however, that the formation of spindles of different sizes can be induced by mechanical perturbations, without any changes in their number or large positional rearrangements of chromosomes (Fig. 1c). Therefore, these chemical signals may either be reset in response to mechanical perturbations, or may partly control the spindle size. On the other hand, the balance in forces generated by molecular motors that push and pull microtubules is also believed to be important for controlling the spindle size ${ }^{18}$. The time scale for structural reorganization of the spindle after the mechanical perturbation was on the order of several minutes, which is comparable to the time scale for mitotic motors to move over micron distances to reorganize microtubule arrangement. Thus, multiple balance points at different sizes may exist for motors that control spindle organization.

\section{Mechanical responses of the spindle function}

Micromanipulation of the mitotic spindle inside a HeLa cell

In complex living tissues and organisms, mitotic cells have to adapt to compressive forces exerted on each other by multiple surrounding cells, in order to carry out cell division safely. It has been demonstrated that the mechanical force produced inside and outside a mitotic cell plays an important role in cellular functions such as changes in cell morphology and/or the determination of spindle orientation via cytoskeleton ${ }^{19-21}$. However, how exactly is the process of chromosome segregation affected by the externally applied force? To directly address this issue, we applied external force to a mitotic spindle in a mammalian cell by mechanical perturbation and examined the response of chromosome segregation machinery ${ }^{22}$, such as the timing of chromosome segregation. As mentioned above, persistent application of force to mitotic spindle induce the reorganization of the spindle structure, even that inside an intact cell ${ }^{11}$. Mitotic HeLa cell, in which all chromosomes were aligned at metaphase plate, was momentarily compressed (ca. $100 \mathrm{msec}$ ) by a pair of cantilevers. As a result, in the case of compression along the spindle pole-to-pole axis, the spindle length (the distance between spindle poles) temporarily shortened, whereas the distance between centromeres (sister chromatids) decreased. On the other hand, the compression applied perpendicular to the pole-to-pole axis resulted in an increase in both spindle length and centromere distance. In addition, the changes in spindle length and centromere distance varied with the extent and loading velocity of the mechanical perturbations. Thus, mechanical perturbations enable us to modulate tension balance within the mitotic spindle inside an intact cell, via cell membrane.

\section{Mechanical responses of a metaphase HeLa cell}

After the completion of chromosome alignment towards the metaphase plate, the correct biorientation of chromosomes inactivates the spindle assembly checkpoint, which produces a wait-anaphase signal to prevent the degradation of mitotic proteins (Cyclin $\mathrm{B}^{23}$ and Securin ${ }^{24}$, Fig. 2a). Chromosome segregation starts just after the cohesions between sister chromatids are cleaved by the cohesin protease (Separase). It is known that the bipolar attachment of kinetochore microtubules is essential for inactivation of spindle assembly checkpoint ${ }^{25}$. On the other hand, microtubule drugs such as taxol or nocodazole have so far been used mainly for testing the influence of tension acting within the mitotic spindle on spindle assembly checkpoint. R. B. Nicklas directly manipulated single chromosome in an intact cell to physically apply tension to it, and proposed that tension is also important to inactivate the spindle assembly checkpoint ${ }^{26}$. In addition, it was reported that the applied tension changed the molecular dynamics of kinetochores (localization and phosphorylation/dephosphorylation of kinetochore proteins) implicated in the mitotic progression ${ }^{27,28}$. Thus, the metaphase progression is considered to be highly sensitive to changes in tension. However, it remains debated whether tension balance in the mitotic spindle determines the dynamics of spindle assembly checkpoint ${ }^{25}$.

Using a microscopic technique of the precisely controlled mechanical perturbations produced by pair of cantilevers, we demonstrated that a short compressive mechanical impulse causes either acceleration or deceleration of the transition from metaphase to anaphase, depending on whether it produces an increase or decrease in tension. Specifically, the acceleration of timing of chromosome segregation was greatly enhanced depending on the extent of an increase in tension. However, these effects were not observed for the case of proper metaphase cells in which chromosomes were aligned at the metaphase plate and the astral microtubules were depolymerized by the application of low doses of nocodazole. Also, mechanical impulses did not facilitate chromosome segregation in prometaphase cells in which unaligned chromosome(s) were present. These results indicate that the applied force is efficiently transmitted to the mitotic spindle inside a cell, plausibly via astral micro- 


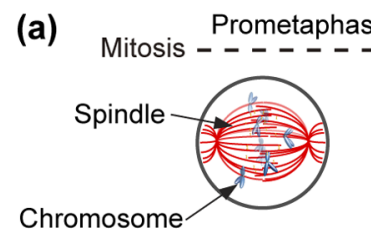

(b)

Chromosome alignment
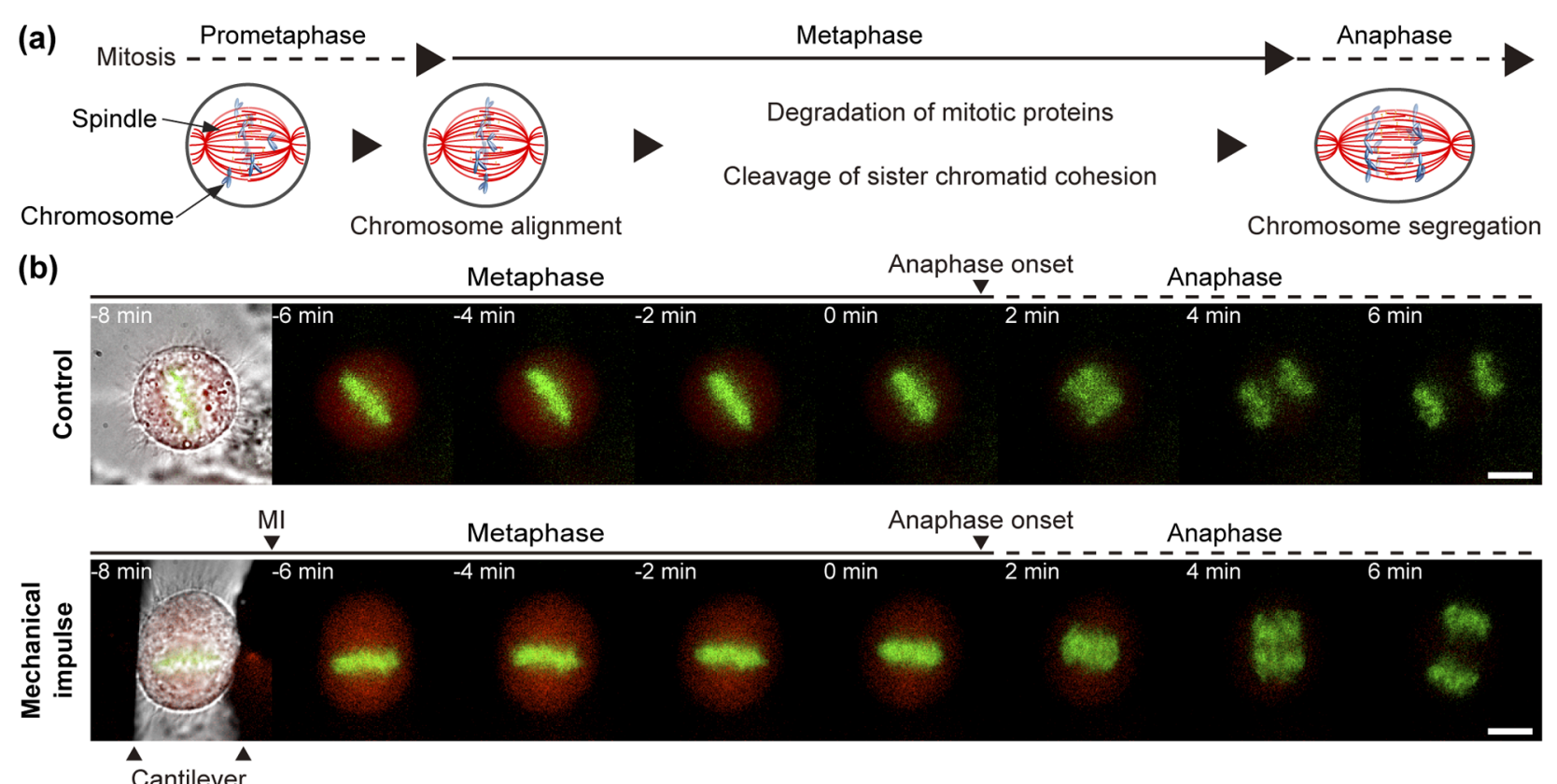

(c)

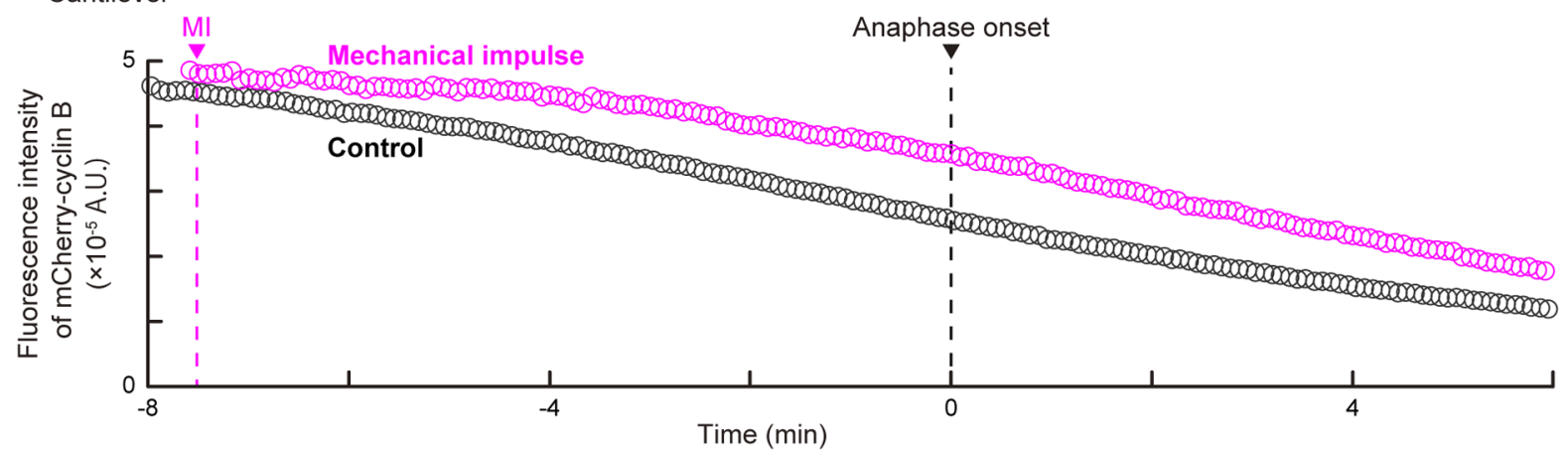

(d) Pole-to-pole compression
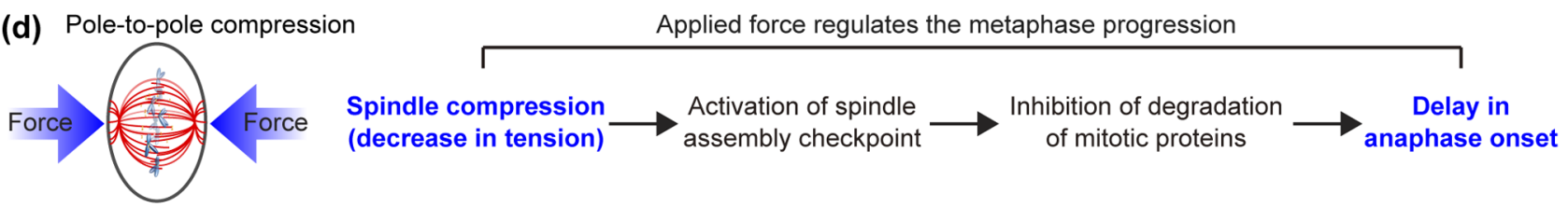

(e) Width compression
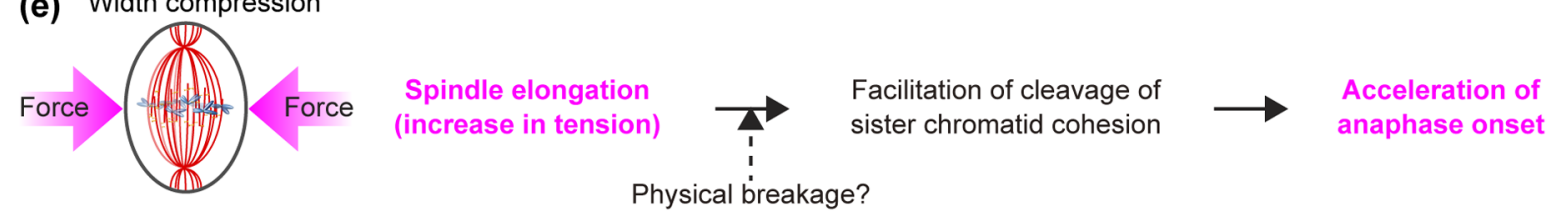

Figure 2 Mechanochemical regulation due to the application of directional mechanical impulse. (a) Schematic illustration of metaphase progression in a mitotic cell. (b) Sequential images of HeLa cells without (top) or with (bottom) the application of mechanical impulse (MI) in the tension-increasing direction, i.e., perpendicular to the pole-to-pole axis. Time 0 indicates the timing of anaphase onset. Chromosomes are shown in green, and mCherry-cyclin B in red. Black regions on the sides of the mitotic cell are the two cantilevers. Scale bars, $10 \mu \mathrm{m}$. (c) Time courses of the change in fluorescence intensity of mCherry-cyclin B in control (black) or MI-applied (magenta) cells, shown in (b). (d, e) Schematic summary describing the mechanochemical regulation of anaphase onset (the timing of chromosome segregation) after the application of mechanical perturbation in the tension-decreasing (d) and the tension-increasing (e) directions.

tubules linked to the cell membrane, which resulted in the subsequent mechanochemical regulation specific to metaphase progression.

How exactly were the molecules, which regulate metaphase progression, mechanochemically affected by mechan- ical perturbation? To probe the details of a mechanochemical regulation in metaphase-anaphase transition, we examined the kinetics of degradation of mitotic proteins (Cyclin B) and dynamics of spindle assembly checkpoint. Degradation of Cyclin B was quantitatively determined by a 
decrease in the fluorescence intensity of mCherry-Cyclin B, which is stably expressed in HeLa cells (Fig. 2b, c) ${ }^{29}$. A decrease in the internal tension due to mechanical perturbation resulted in the deceleration of degradation in mitotic proteins and the delay of anaphase onset. As described above, the degradation of mitotic proteins is closely related to the inhibition of spindle assembly checkpoint. The activation of spindle assembly checkpoint immediately arrests the metaphase progression even after the mitotic protein degradation has started ${ }^{23}$. The analysis of the dynamics of spindle checkpoint proteins, such as BubR1 and Mad2, showed that only a decrease in tension induced the accumulation of EGFP-BubR1 to the kinetochore, which is generally accepted as a monitor showing the deficient tension on kinetochores ${ }^{30}$. On the other hand, Mad2, which senses errors in the kinetochore-microtubule attachment ${ }^{31}$, was not detected after cells were compressed in any direction. These results indicate that the mechanical perturbation, which decreases tension along the spindle pole-to-pole axis, induces the activation of spindle assembly checkpoint, such that the degradation of mitotic proteins is decelerated, which results in a delay in the timing of chromosome segregation (Fig. 2d). Thus, spindle assembly checkpoint has a role to prevent errors in chromosome segregation by detecting the defects in the microtubule attachment and/or tension on kinetochores, and is the surveillance system to lead the conversion of mechanical force to chemical signal.

On the other hand, the mechanical perturbation in the tension-increasing direction did not induce the localization of checkpoint proteins to kinetochores and greatly accelerated chromosome segregation. These results suggest that the transient excess of tension is not a potential source for spindle assembly checkpoint to produce a wait-anaphase signal. Change in kinetics of cyclin B degradation was not observed, but chromosome segregation occurred even when cyclin $\mathrm{B}$ was only partly degraded (Fig. 2c). Although mitotic cells treated with a proteasome inhibitor never undergo anaphase, due to the inhibition of degradation of mitotic proteins, mechanical perturbation could trigger the chromosome segregation. Therefore, a reasonable interpretation is that the mechanical perturbation can increase the force imposed on each sister chromatid cohesion as the mitotic spindle stretches, which results in the physical breakage of a part of the sister chromatid cohesion (Fig. 2e).

\section{Perspectives}

The results obtained from the mechanical perturbations demonstrate that chromosome segregation machinery mechanochemically responds to the external applied force in the direction- and magnitude-dependent manner, whereas it could also depend on temporal variation of the external force. As we described above, the persistent application of the external force over a few minutes produced the structural adaptation of the metaphase spindle assembled in vitro.
On the other hand, the millisecond-order mechanical impulse modulated the timing of chromosome segregation in a mammalian cell. The cell division and the organization of its hierarchical machinery should be performed flawlessly even when the external forces are applied over short and long time scales. Our results suggest that properly applied force alone is able to assist chromosome segregation in living cells to occur at the right time and place.

Physical forces generated inside or outside the cells affect local mechanical properties and cellular behavior ${ }^{19,32}$. Different types of cells with various mechanical properties may be specifically suited to produce particular responses to the external force ${ }^{33}$. Mechanical perturbations such as those introduced here mimic the directional external forces exerted by the adjacent cells on each other in the complex systems composed of multiple cells. Mechanical interactions between cells could be one of the regulators not only in cell division, but also in gene expression patterns and the hierarchical organization ${ }^{34-36}$. Future works using quantitative microscopic (or nanoscopic) techniques will provide new insights into how the mechanical interactions, which are intricately involved in cellular processes both mechanically and biochemically, have an essential role in the destination of cells.

\section{Acknowledgements}

We gratefully acknowledge Dr. Tarun M. Kapoor (Rockefeller University), Dr. Yasuhiko Terada (Waseda University), and Dr. Isao Shimoyama (The University of Tokyo) for collaborative studies described here. The work in our laboratory was supported in part by Grants-in-Aid for Scientific Research on Priority Areas (to T.I.), Grants-inAid for Scientific Research (C) (to T. I.), Research Fellowship for Young Scientists (DC1) (to J. T. and K. S.), Grantsin-Aid for Specially Promoted Research, Scientific Research (S) (to S. I.), and a Research Grant from the Human Frontier Science Program (to S. I.).

\section{References}

1. Mitchison, T. J. \& Salmon, E. D. Mitosis: a history of division. Nat. Cell Biol. 3, E17-21 (2001).

2. Neumann, B., Walter, T., Hériché, J. K., Bulkescher, J., Erfle, H., Conrad, C., Rogers, P., Poser, I., Held, M., Liebel, U., Cetin, C., Sieckmann, F., Pau, G., Kabbe, R., Wünsche, A., Satagopam, V., Schmitz, M.H., Chapuis, C., Gerlich, D. W., Schneider, R., Eils, R., Huber, W., Peters, J.M., Hyman, A. A., Durbin, R., Pepperkok, R. \& Ellenberg, J. Phenotypic profiling of the human genome by time-lapse microscopy reveals cell division genes. Nature 464, 721-727 (2010).

3. Hutchins, J. R., Toyoda, Y., Hegemann, B., Poser, I., Hériché, J.K., Sykora, M.M., Augsburg, M., Hudecz, O., Buschhorn, B. A., Bulkescher, J., Conrad, C., Comartin, D., Schleiffer, A., Sarov, M., Pozniakovsky, A., Slabicki, M. M., Schloissnig, S., Steinmacher, I., Leuschner, M., Ssykor, A., Lawo, S., Pelletier, L., Stark, H., Nasmyth, K., Ellenberg, J., Durbin, R., Buchholz, 
F., Mechtler, K., Hyman, A.A. \& Peters, J.M. Systematic analysis of human protein complexes identifies chromosome segregation proteins. Science 328, 593-599 (2010).

4. McIntosh, J.R., Molodtsov, M. I. \& Ataullakhanov, F. I. Biophysics of mitosis. Q. Rev. Biophys. 45, 147-207 (2012).

5. Hiramoto, Y. Mechanical properties of the protoplasm of the sea urchin egg. II. Fertilized egg. Exp. Cell Res. 56, 209-218 (1969).

6. Stewart, M.P., Helenius, J., Toyoda, Y., Ramanathan, S.P., Muller, D. J. \& Hyman, A. A. Hydrostatic pressure and the actomyosin cortex drive mitotic cell rounding. Nature 469, 226-230 (2011).

7. Nicklas, R.B. Measurements of the force produced by the mitotic spindle in anaphase. J. Cell Biol. 97, 542-548 (1983).

8. Hiramoto, Y. \& Nakano, Y. Micromanipulation studies of the mitotic apparatus in sand dollar eggs. Cell Motil. Cytoskeleton 10, 172-184 (1988)

9. Itabashi, T., Takagi, J., Shimamoto, Y., Onoe, H., Kuwana, K., Shimoyama, I., Gaetz, J., Kapoor, T. M. \& Ishiwata, S. Probing the mechanical architecture of the vertebrate meiotic spindle. Nat. Methods 6, 167-172 (2009).

10. Shimamoto, Y., Maeda, Y. T., Ishiwata, S., Libchaber, A. J. \& Kapoor, T. M. Insights into the micromechanical properties of the metaphase spindle. Cell 145, 1062-1074 (2011).

11. Dumont, S. \& Mitchison, T. J. Compression regulates mitotic spindle length by a mechanochemical switch at the poles. Curr. Biol. 19, 1086-1095 (2009).

12. Kalab, P., Weis, K. \& Heald, R. Visualization of a Ran-GTP gradient in interphase and mitotic Xenopus egg extracts. Science 295, 2452-2456 (2002)

13. Caudron, M., Bunt, G., Bastiaens, P. \& Karsenti, E. Spatial coordination of spindle assembly by chromosome-mediated signaling gradients. Science 309, 1373-1376 (2005).

14. Andersen, S.S., Ashford, A.J., Tournebize, R., Gavet, O., Sobel, A., Hyman, A. A. \& Karsenti, E. Mitotic chromatin regulates phosphorylation of Stathmin/Op18. Nature 389, 640-643 (1997).

15. Ohi, R., Burbank, K., Liu, Q. \& Mitchison, T. J. Nonredundant functions of Kinesin-13s during meiotic spindle assembly. Curr. Biol. 17, 953-959 (2007).

16. Loughlin, R., Wilbur, J.D., McNally, F. J., Nedelec, F. J. \& Heald, R. Katanin contributes to interspecies spindle length scaling in Xenopus. Cell 147, 1397-1407 (2011).

17. Karsenti, E. \& Vernos, I. The mitotic spindle: a self-made machine. Science 294, 543-547 (2001).

18. Hildebrandt, E. R. \& Hoyt, M. A. Mitotic motors in Saccharomyces cerevisiae. Biochim. Biophys. Acta 1496, 99-116 (2000).

19. Effler, J.C., Kee, Y. S., Berk, J. M., Tran, M. N., Iglesias, P. A. \& Robinson, D. N. Mitosis-specific mechanosensing and contractile-protein redistribution control cell shape. Curr. Biol. 16, 1962-1967 (2006).

20. Fink, J., Carpi, N., Betz, T., Betard, A., Chebah, M., Azioune, A., Bornens, M., Sykes, C., Fetler, L., Cuvelier, D. \& Piel, M.
External forces control mitotic spindle positioning. Nat. Cell Biol. 13, 771-778 (2011).

21. Minc, N., Burgess, D. \& Chang, F. Influence of cell geometry on division-plane positioning. Cell 144, 414-426 (2011).

22. Itabashi, T., Terada, Y., Kuwana, K., Kan, T., Shimoyama, I. \& Ishiwata, S. Mechanical impulses can control metaphase progression in a mammalian cell. Proc. Natl. Acad. Sci. USA 109, 7320-7325 (2012).

23. Clute, P. \& Pines, J. Temporal and spatial control of cyclin B1 destruction in metaphase. Nat. Cell Biol. 1, 82-87 (1999).

24. Hagting, A., Den Elzen, N., Vodermaier, H.C., Waizenegger, I. C., Peters, J.M. \& Pines, J. Human securin proteolysis is controlled by the spindle checkpoint and reveals when the APC/C switches from activation by $\mathrm{Cdc} 20$ to Cdh1. J. Cell Biol. 157, 1125-1137 (2002).

25. Maresca, T. J. \& Salmon, E. D. Welcome to a new kind of tension: translating kinetochore mechanics into a wait-anaphase signal. J. Cell Sci. 123, 825-835 (2010).

26. Li, X. \& Nicklas, R. B. Mitotic forces control a cell-cycle checkpoint. Nature 373, 630-632 (1995).

27. Nicklas, R. B., Campbell, M. S., Ward, S. C. \& Gorbsky, G. J. Tension-sensitive kinetochore phosphorylation in vitro. J. Cell Sci. 111, 3189-3196 (1998).

28. Nicklas, R. B., Ward, S. C. \& Gorbsky, G. J. Kinetochore chemistry is sensitive to tension and may link mitotic forces to a cell cycle checkpoint. J. Cell Biol. 130, 929-939 (1995).

29. Uchida, K.S., Takagaki, K., Kumada, K., Hirayama, Y., Noda, T. \& Hirota, T. Kinetochore stretching inactivates the spindle assembly checkpoint. J. Cell Biol. 184, 383-390 (2009).

30. Elowe, S., Hummer, S., Uldschmid, A., Li, X. \& Nigg, E. A. Tension-sensitive Plk1 phosphorylation on BubR1 regulates the stability of kinetochore microtubule interactions. Genes Dev. 21, 2205-2219 (2007).

31. Waters, J.C., Chen, R.H., Murray, A.W. \& Salmon, E.D. Localization of Mad2 to kinetochores depends on microtubule attachment, not tension. J. Cell Biol. 141, 1181-1191 (1998).

32. Discher, D. E., Janmey, P. \& Wang, Y. L. Tissue cells feel and respond to the stiffness of their substrate. Science 310, 1139$1143(2005)$

33. Discher, D. E., Mooney, D. J. \& Zandstra, P. W. Growth factors, matrices, and forces combine and control stem cells. Science 324, 1673-1677 (2009).

34. Cheng, G., Tse, J., Jain, R.K. \& Munn, L.L. Microenvironmental mechanical stress controls tumor spheroid size and morphology by suppressing proliferation and inducing apoptosis in cancer cells. PLoS One 4, e4632 (2009).

35. Kuroda, R., Endo, B., Abe, M. \& Shimizu, M. Chiral blastomere arrangement dictates zygotic left-right asymmetry pathway in snails. Nature 462, 790-794 (2009).

36. Engler, A. J., Sen, S., Sweeney, H. L. \& Discher, D. E. Matrix elasticity directs stem cell lineage specification. Cell 126, 677-689 (2006). 